\title{
Review Article \\ Recent Advances on SEM-Based In Situ Multiphysical Characterization of Nanomaterials
}

\author{
Juntian Qu $\mathbb{D}^{1,2,3}$ and Xinyu Liu $\mathbb{D D}^{4}$ \\ ${ }^{1}$ State Key Laboratory of Tribology \& Institute of Manufacturing Engineering, Department of Mechanical Engineering, \\ Tsinghua University, Beijing 100084, China \\ ${ }^{2}$ Beijing Key Laboratory of Precision/Ultra-Precision Manufacturing Equipments and Control, Tsinghua University, \\ Beijing 100084, China \\ ${ }^{3}$ Department of Mechanical Engineering, McGill University, Montreal, H3A 0G4, Canada \\ ${ }^{4}$ Department of Mechanical and Industrial Engineering, University of Toronto, Toronto, M5S 3G8, Canada
}

Correspondence should be addressed to Juntian Qu; juntian.qu@mail.mcgill.ca

Received 7 April 2021; Revised 18 May 2021; Accepted 22 May 2021; Published 9 June 2021

Academic Editor: Nicolas Delorme

Copyright (C) 2021 Juntian Qu and Xinyu Liu. This is an open access article distributed under the Creative Commons Attribution License, which permits unrestricted use, distribution, and reproduction in any medium, provided the original work is properly cited.

\begin{abstract}
Functional nanomaterials possess exceptional mechanical, electrical, and optical properties which have significantly benefited their diverse applications to a variety of scientific and engineering problems. In order to fully understand their characteristics and further guide their synthesis and device application, the multiphysical properties of these nanomaterials need to be characterized accurately and efficiently. Among various experimental tools for nanomaterial characterization, scanning electron microscopy- (SEM-) based platforms provide merits of high imaging resolution, accuracy and stability, well-controlled testing conditions, and the compatibility with other high-resolution material characterization techniques (e.g., atomic force microscopy), thus, various SEM-enabled techniques have been well developed for characterizing the multiphysical properties of nanomaterials. In this review, we summarize existing SEM-based platforms for nanomaterial multiphysical (mechanical, electrical, and electromechanical) in situ characterization, outline critical experimental challenges for nanomaterial optical characterization in SEM, and discuss potential demands of the SEM-based platforms to characterizing multiphysical properties of the nanomaterials.
\end{abstract}

\section{Introduction}

The last two decades have witnessed the extensive research on nanomaterials because of their exceptional promise in science and technology. Based on structural dimension, existing nanomaterials fall into four categories of nanostructures: zero-dimensional structures (e.g., nanoparticles, nanospheres, and isolated molecules) [1], one-dimensional structures (e.g., nanowires, nanobelts, nanotubes, and nanoribbons) [2-4], two-dimensional structures (e.g., nanofilms, grapheme, and molybdenum disulfide) $[5,6]$, and three-dimensional structures (e.g., nanocombs, nanoflowers, and nanocups) [7-9]. Due to their superior physical properties and unique nanoscale morphologies, these nanomaterials have been widely used for a variety of applications such as next-generation elec- tronics [10], sustainable energy [11], biosensing [12], and (opto) electronics [13]. The mechanical, electrical, and optical properties of these nanomaterials play critical roles in their practical uses, and the experimental determination of these properties is thus of major concern from the perspective of both nanomaterial synthesis and applications.

Among various experimental techniques employed for nanomaterial characterization, emerging technique of nanorobotic manipulation in scanning electron microscopy (SEM) has enabled various multiterminal characterization of nanomaterials and nanostructures, such as electrical and mechanical measurements [14, 15]. On one hand, nanomanipulation has filled the gap between top-down and bottom-up approaches and realized position control at the nanometer scale [16] and provides effective strategy for the property 
characterization of individual nanoscale materials and the construction of nanoscale devices [16]. On the other hand, SEM can provide real-time imaging with nanometer resolution and a large scanning area, which enables the development and integration of robotic nanomanipulation systems inside large vacuum chamber to realize simultaneous imaging and direct interactions with objects at the submicrometer and nanometer scales [14]. In addition, SEM can also be integrated with the latest technology (e.g., electron beam lithography (EBL) and focused ion beam (FIB)) to perform in situ nanomaterial engineering and fabrication [17].

Benefiting from above merits, the combination of nanomanipulation technique and SEM has extended both our eyes and hands simultaneously to nanoscale providing an intuitionistic, real-time, and in situ way to study nanomaterials and perform nanomaterial characterization in SEM [15]. However, due to existing challenges in the optics integration into SEM, most nanomaterial in situ characterization techniques in SEM are limited in mechanical, electrical, and electromechanical measurements; few work has been reported for optical characterization of nanomaterials.

This review presents a survey of recent advances in in situ multiphysical characterization of nanomaterials in SEM, including mechanical, electrical, and electromechanical characterization. Challenges and limitations of the optical characterization in SEM are analyzed, and prospects for multiphysical nanomaterial characterization are also discussed.

\section{In Situ Multiphysical Characterization in SEM}

Regarding the topic of SEM-based nanomaterial characterization, there are some several reviews in the literature. Fukuda et al. [18] reviewed the assembly of nanodevice and the in situ property characterization of carbon nanotubes through nanorobotic manipulation. Shi et al. [14] also reviewed the applications of nanorobotic manipulation in the characterization of nanomaterials and nanostructures. Haque et al. [19] and Zhu et al. [20] reviewed the recent advances in MEMS-based devices for nanomechanical characterization. Besides, Fukuda et al. [21] and Shen et al. [22] reviewed the advanced applications of micronanorobotic manipulation on single-cell analysis and characterization in ESEM. Jiang et al. [23] reviewed the recent advances on in situ SEM mechanical and electrical characterization of lowdimensional nanomaterials; the common electromechanical characterization methods of piezoelectric one-dimensional materials were reviewed by Majid et al. [24] as well.

In the following sections, we will focus our review on the topic of in situ multiphysical characterization of nanomaterials in SEM, including mechanical, electrical, and electromechanical fields-coupled characterization. Different from the previous review [14] focusing on nanorobotic manipulation systems, we will mainly discuss the characterization methodologies based on different testing types (experimental setups) in each field. In the meanwhile, we will analyze the current status of optical-measurement-related nanomaterial characterization in SEM.
2.1. Mechanical Characterization in SEM. The understanding of mechanical properties of nanomaterials plays important roles in miniaturized electronic, optical, thermal, and electromechanical systems. However, due to the scaling effects and geometric differences, when the surface-to-volume ratio increases along with the decreased size of structures, nanostructures such as nanowires (NWs), carbon nanotubes (CNTs), and ultrathin films tend to exhibit significantly different mechanical properties compared with their bulk counterparts [25-27], which means that we cannot easily deduce nanomaterial mechanical properties from bulk properties. Besides, the well-established techniques for mechanical characterization at macroscale cannot be totally transplanted to nanoscale in the respect of equipment and resolution limitations [28]. SEM-based nanomanipulation deals with above challenges for mechanical characterization of nanomaterials in various ways, which are summarized and classified by the testing type (Table 1).

2.1.1. SEM-Based In Situ Mechanical Characterization. In situ bending test was performed on individual multiwalled carbon nanotubes (MCNTs) to characterize its Young's modulus, where the bending force was detected by a piezoresistive atomic force microscope (AFM) probe [29], as shown in Figure 1(a). Also, the individual MCNT's Young's modulus can be determined by in situ buckling test $[18,30]$, as shown in Figure 1(b). An individual MCNT was EBIDfixed with AFM cantilever probe via nanomanipulation, and the buckling force was measured by the deflection of cantilever beam [18].

In situ tensile test [31] was employed to study the strength and breaking mechanism of MCNTs, as shown in Figure 1(c). An individual MCNT was EBID-mounted between two opposing AFM tips with different cantilever stiffness, the upper rigid cantilever was driven upward to apply tensile load to the MCNT, and the tensile force was determined by tracking the deflection of the lower soft cantilever [31]. With a similar principle, mechanical characterization of InGaAs/GaAs nanosprings [32], Si nanowires $[33,34]$, and Ag nanowires [35] was also performed by in situ tensile tests, as shown in Figure 1(d). Accurate strain measurements based on high-resolution SEM imaging of $\mathrm{Ag}$ NWs facilitated the acquisition of full spectrum of mechanical properties including Young's modulus, yield strength, and ultimate tensile strength [35].

Besides above testing methods, for fragile twodimensional materials, as a nondestructive assessment method, in situ nanoindentation measurements have been employed to examine the mechanical properties of a fewlayer graphene membrane [36], individual graphene flakes [37] (Figure 1(e)), and nanopaper made of microfibrillated cellulose [38] (Figure 1(f)), where characteristic forcedisplacement curves were recorded during the indentation process to extract the Young's modulus. Also, local stiffness of InP suspended micromembrane was first-time measured by a tuning-fork-based dynamic force sensor inside SEM [39]. The surface mechanical properties of low-density polyethylene (LDPE) reinforced by carbon nanofibers (CNFs) were investigated using nanoindentation as well [40]. 
TABLE 1: Mechanical characterization of nanomaterials in SEM.

(a)

\begin{tabular}{lccc}
\hline Testing types & Nanomaterials & Properties & References \\
\hline Bending & MCNTs & Young's modulus & {$[29]$} \\
\hline Buckling & MCNTs & Young's modulus & {$[18,30]$} \\
\hline & MCNTs & Strength and breaking mechanism & {$[31]$} \\
Tensile & InGaAs/GaAs nanosprings & Stiffness & {$[32]$} \\
& Si nanowires & Yield strength & {$[33,34]$} \\
& Ag nanowires & Yield, ultimate tensile strength & {$[35]$} \\
\hline & Graphene membrane & Elastic stiffness and Young's modulus & {$[36]$} \\
Nanoindentation & Graphene flakes & Young's modulus & {$[37]$} \\
& Nanopaper & Young's modulus & {$[38]$} \\
& InP membranes & Local stiffness & {$[39]$} \\
\hline
\end{tabular}

(b) ESEM for biological cells

\begin{tabular}{|c|c|c|c|}
\hline Nanomaterials & Properties & End-effectors & References \\
\hline \multirow{4}{*}{ Wild type yeast cells } & Single cell adhesion force & Nanofork & {$[46,47]$} \\
\hline & Cell-cell adhesion force & Nanopicker & {$[48]$} \\
\hline & Cell-surface adhesion force & Flat AFM cantilever tips & {$[49,50]$} \\
\hline & Stiffness, viscoelastic properties & Soft buckling nanoneedles & {$[51-55]$} \\
\hline Microbead and biological cell & Cell detachment force & FIB etched AFM cantilever & [56] \\
\hline
\end{tabular}

Except for the abovementioned cases where mechanical properties were in situ characterized in SEM vacuum chamber, there are also certain scenarios where SEM was employed as critical tool for surface morphology imaging, which could assist the following mechanical characterization of nanomaterials/nanostructures. Gantayat et al. [41] wrote a review on carbon nanomaterial-reinforced epoxy composites; based on the summary in this review, SEM was proved to be an extremely useful tool to investigate the microstructure and surface morphology of many nanomaterials; moreover, SEM-based results could provide important information for correlated mechanical characterization process [42]. Specifically, the evaluation of surface modification impact on PP/MWCNT nanocomposites by mechanical characterization was assisted with SEM morphological image processing [43]. Similarly, the surface micrographs of nanocomposite films were procured by SEM to explore the nanoparticles' dispensation [44], assisting the demonstration of greater mechanical properties (high tensile strength and Young's modulus). What is more, SEM was also used to characterize the phase separation process in thin electrical conductive composite (ECC) films mixed with nanostructured polyaniline (PANI) [45], proving its enhanced mechanical durability.

2.1.2. ESEM-Based In Situ Mechanical Characterization of Biological Cells. For cell mechanical characterization in Environmental SEM (ESEM), standard AFM cantilevers were modified using FIB etching and deposition to produce differ- ent types of functional tools [14], such as nanofork [46], nanopicker [48], soft buckling nanoneedles [51-55], and flat AFM cantilever tips $[49,50]$, as shown in Figures 2(a)-2(c). These customized end-effectors were mounted onto the nanomanipulation system in ESEM for indentation to in situ characterize the stiffness $[51,52,55]$ and viscoelastic properties [53] of single cells (Figure 2(d)), as well as the mechanical properties of individual yeast cells [49] and cell nucleus [54].

Besides, cell-surface adhesion force is important for cell activities and the development of biomaterials, and an in situ cell force measurement system was developed based on nanorobotic manipulation inside an ESEM (Figure 2(e)) to characterize the single cell adhesion force [46, 47], cellsurface adhesion force [50], and cell-cell adhesion force [48]. Shen et al. [56] also developed a dynamic force characterization system to investigate the cell detachment process at small scales.

2.1.3. MEMS-Based In Situ Mechanical Characterization in SEM. The MEMS-based tensile testing has been applied to characterizing 1D nanostructures (e.g., nanowires, nanotubes, and nanoribbons) and can measure the sample's mechanical properties such as Young's modulus, failure strain and fracture strength [57]. In a tensile test, a 1D nanosample is mounted across a micrometer-sized gap on a MEMS device, and an on-chip microactuator stretches the sample from one side of the gap, and a microforce sensor measures the tension force of the sample on the other side of the gap. The elongation (thus tension strain) of the sample 


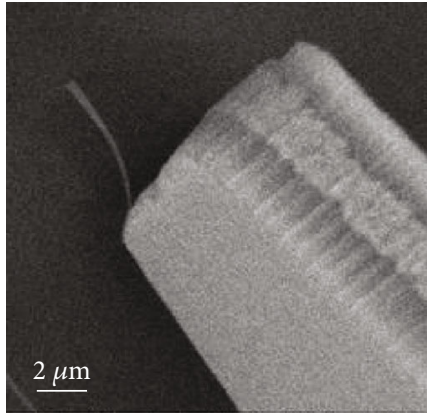

(a)

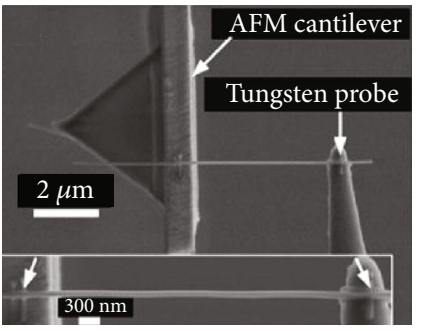

(d)

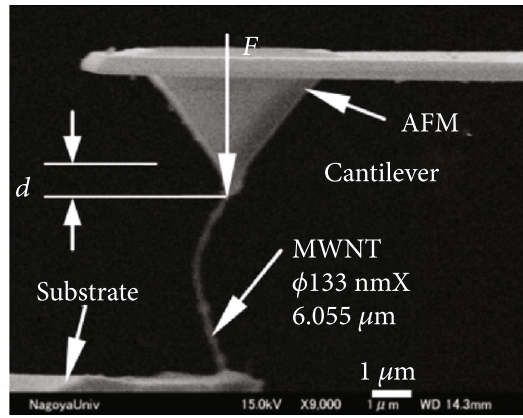

(b)

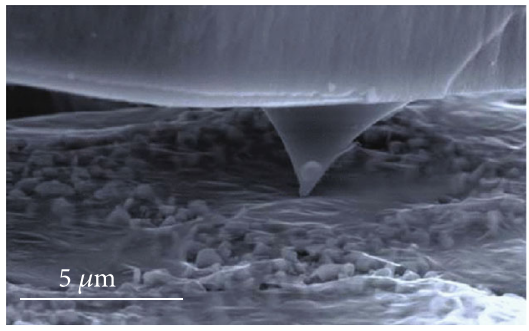

(e)

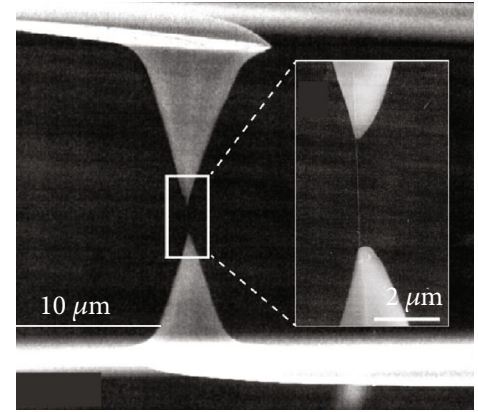

(c)

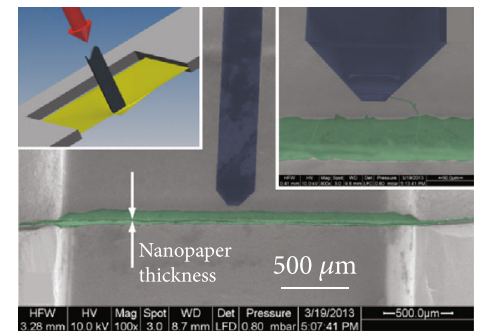

(f)

FIGURE 1: SEM-based in situ mechanical characterization of nanomaterials. (a) Deflection of an individual multiwalled carbon nanotube (MWCNT) using a piezoresistive atomic force microscope (AFM). (b) In situ mechanical characterization of a nanotube by buckling test. (c) Tensile test of individual MCNT, inset of (c) shows the enlarged view of MCNT under tensile force. (d) Tensile test of a single Ag NW, inset of (d) shows a high-resolution SEM image of the NW for strain measurement. (e) The AFM probe is deflecting the graphene flake while measuring the acting forces. (f) Measurement scene of nanopaper inside SEM: the top left inset shows the stretched nanopaper, and the top right inset shows the nanopaper thickness measurement.

can be quantified via high-resolution imaging (using an optical or electron microscope) [58-60] or on-chip measurement of the sample-mounting gap size [61].

Various typical MEMS platforms were developed for tensile test in SEM, taking advantage of its high-resolution realtime imaging capability for in situ observation of material behaviors. Tracing back to the year of 2001, Haque and Saif [62] have proposed the potential application of MEMS actuators on micromechanical testing in an SEM chamber, based on a demonstration of uniaxial tensile test on freestanding thin films in the microsubmicrometer-scale using MEMS devices. The fact of very small overall setup size facilitates the in situ observation of materials behavior in SEM chamber [62]. Zhu et al. [59] reported the development of a material testing system for in situ electron microscopy (EM) mechanical testing of nanostructures and demonstrated in situ EM testing of free-standing polysilicon films, metallic nanowires, and carbon nanotubes. Espinosa et al. [63] developed the first MEMS-based material testing scheme that can continuously observe specimen deformation with subnanometer resolution and simultaneously measure tension force with nanoNewton resolution. B. Pant et al. [58] proposed a versatile MEMS material testing setup that supports both in situ and ex situ testing of nanomaterial with high accuracy and precision. Except for the abovementioned work, there are also MEMS material testing systems for characterizing 2D nanoscale films $[64,65]$ and $1 D$ nanomaterials $[66,67]$.

Among various types of nanomaterials in situ characterized by MEMS-based tensile platforms, carbon nanotube (CNT) is the representative one. The mechanical properties of CNT were characterized by various in situ MEMS platforms in SEM: as shown in Figure 3(a), in situ tensile loading of a templated carbon nanotube (T-CNT) was reported in [68], where the load was derived from the bending of the direct force-sensing beam and the elongation of the specimen can be obtained from SEM images. Peng et al. [69] employed in situ MEMS tensile tester and exploited the excellent mechanical properties of CNTs with extreme high fracture strength. As shown in Figures 3(b) and 3(a), multiwalled nanotube was bridged between the gap of the actuator (left) and the load sensor. Also, the in situ mechanical characterization of free-standing cofabricated polysilicon films and multiwalled carbon nanotubes (MCNTs) in SEM was employed as a validation MEMS-based material tensile testing system, which for the first time achieved continuous observation of the specimen deformation and load measurement electronically with nano-Newton resolution [63]. Zhu et al. $[59,70]$ designed a MEMS device for the tensile testing of CNTs with two types of actuators: thermal and electrostatic actuators. The device with a thermal actuator [59] was used for displacement-controlled testing and the one with a comb-drive electrostatic actuator [70] for forcecontrolled testing.

Except for CNTs, the mechanical properties of a series of nanowires have also been characterized by in situ MEMS platforms in SEM. Kiuchi et al. [67] employed electrostatic actuated nanotensile testing devices (EANATs) to measure the mechanical properties of single carbon nanowire which is suspended between actuation beams (Figure 3(c)), and the Young's modulus and fracture stress-strain of the 


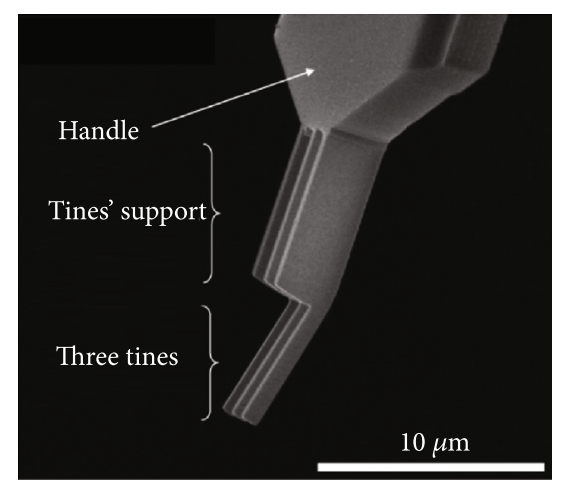

(a)

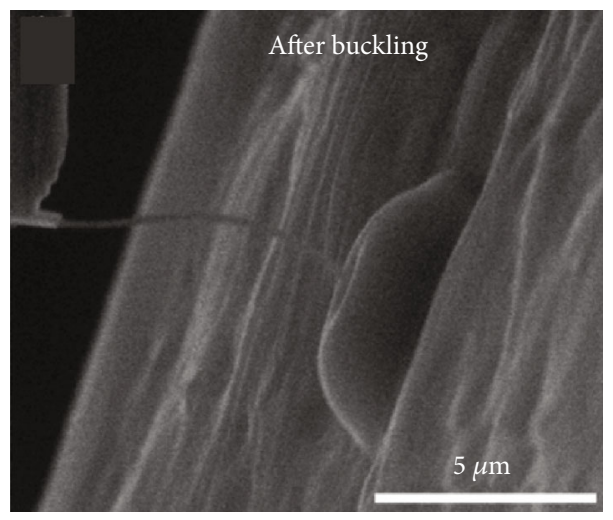

(d)

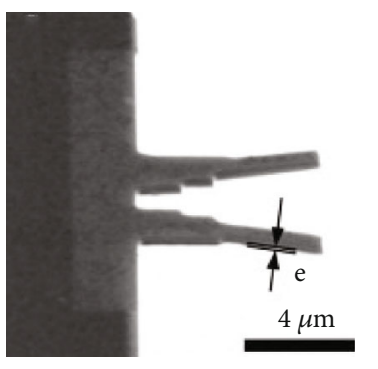

(b)

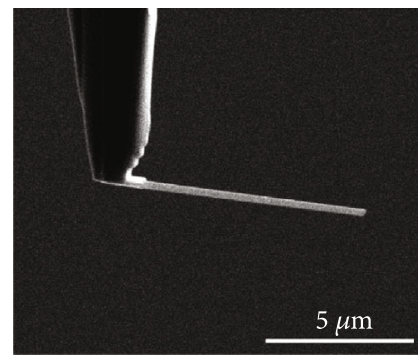

(c)

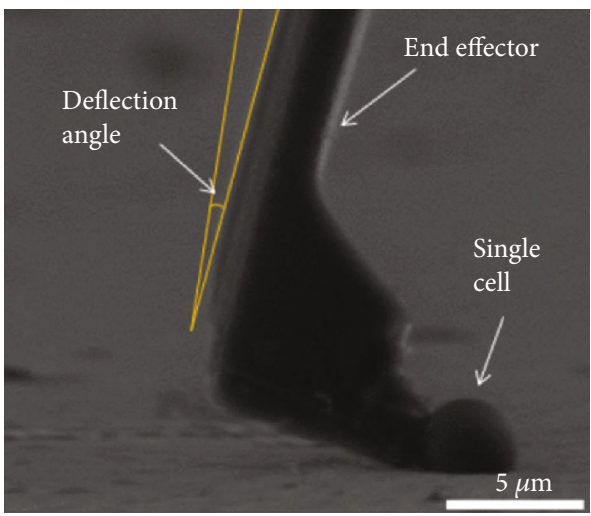

(e)

FIGURE 2: ESEM-based mechanical characterization of biological cells. SEM images of (a) nanofork, (b) nanopicker tip, and (c) soft buckling nanoneedle. (d) Single cell global stiffness measurement using Si nanoneedle in buckling condition. (e) Cell adhesion force measurement.

nanowires were accurately obtained. Zhang et al. [71] performed uniaxial quasi-static tensile testing on individual nanocrystalline Co NWs in SEM using on-chip MEMS tensile testing system consisting of a comb-drive actuator and a clamped-clamped beam force sensor. As an extended work, Zhang et al. [72] further developed two types of electrostatically actuated tensile stages with either a differential capacitive sensor or a clamped-clamped beam force sensor for mechanical characterization of individual Si NWs, as shown in Figure 3(d). Besides, the fracture mechanism of zinc oxide nanowires was investigated under uniaxial tensile loading utilizing a MEMS-based nanoscale material testing stage [73]. Employing thermal actuator, Brown et al. [74] reported direct tensile tests on $\mathrm{n}$-type (Si-doped) gallium nitride single crystal nanowires (GaN NWs), as shown in Figure 3(e), where tensile strength of NWs was characterized and the failure modes were analyzed.

Similar to tensile tests, mechanical properties of nanomaterials can also be evaluated via MEMS-based bend testing, which represents another type of widely used experimental technique [78]. In a typical MEMS-based bend testing setup [78], a cantilever beam (cofabricated with the MEMS device) is moved by a comb-drive electrostatic actuator and bent against a fixed block. Haque and Saif [62] proposed a MEMS-based setup, which employs a comb-drive electrostatic actuator to bend test a $100 \mathrm{~nm}$ thick aluminum cantilever beam. The applied force was calculated using the precalibrated loading equation of the actuator, and the beam deformation was measured via high-resolution imaging. Corigliano et al. [77] proposed a rotary comb-drive actuator and a parallel-plate electrostatic actuator for in-plane and out-of-plane bend testing of thin-film $(700 \mathrm{~nm})$ polysilicon microstructures, respectively. The microstructures were cofabricated via a commercial surface micromachining process, which allows for nanometer-thick polysilicon structures to be attached to the bottom of micrometer-think polysilicon MEMS structures. This cofabrication process eliminates the need for nanosample addition after MEMS device fabrication (which could be technically challenging).

Based on above descriptions and discussions, the MEMSbased mechanical characterization techniques of micro- and nanomaterials are summarized in Table 2 , classified by different testing types and different categories of materials.

2.2. Electrical Characterization in SEM. Better understanding of the electrical properties of nanomaterials will contribute to the development of next-generation nanoelectronics and nanosensors which promise ultrahigh performance [79]. Typically, there are three kinds of methods adopted during in situ electrical characterization of nanomaterials: fourpoint, two-point, and three-point probing.

2.2.1. Four-Point Probing. Four-point measurement is a widely adopted technique to eliminate the effect of contact 


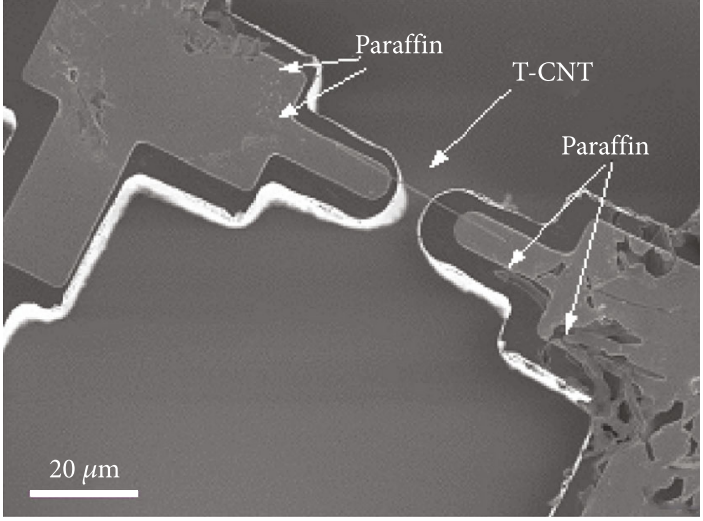

(a)

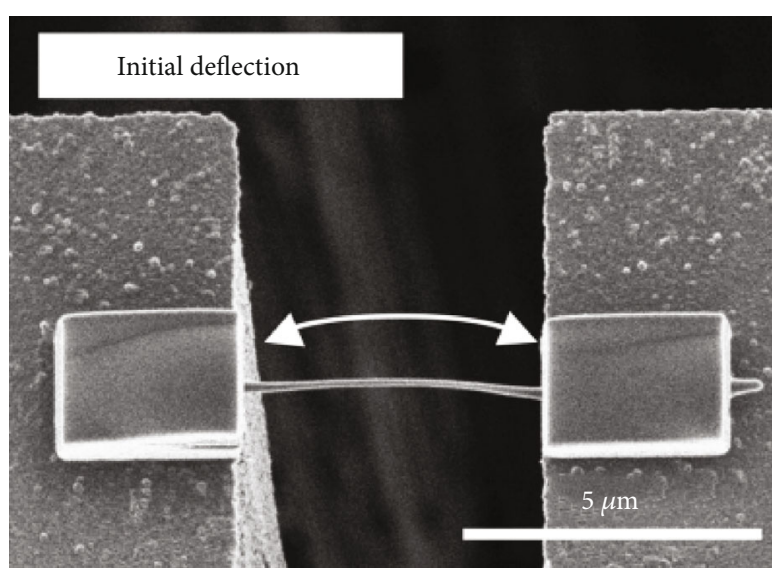

(c)

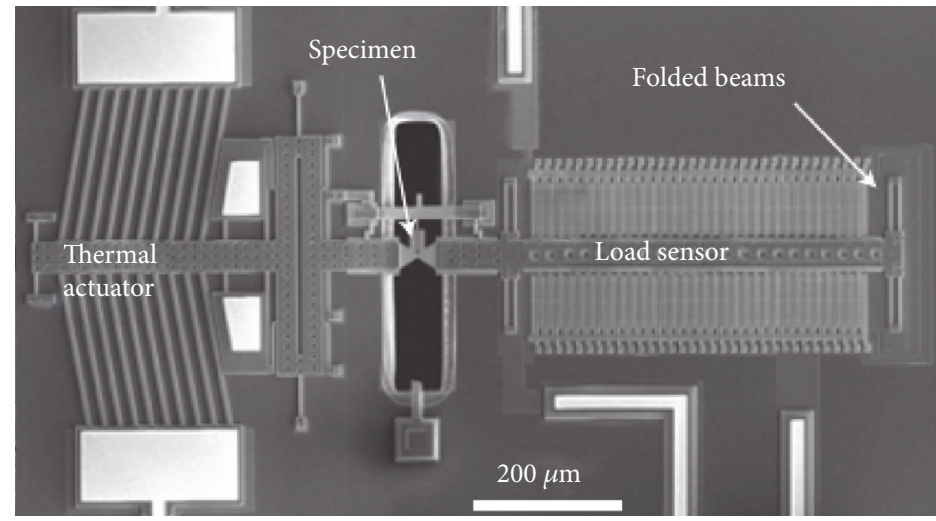

(b)

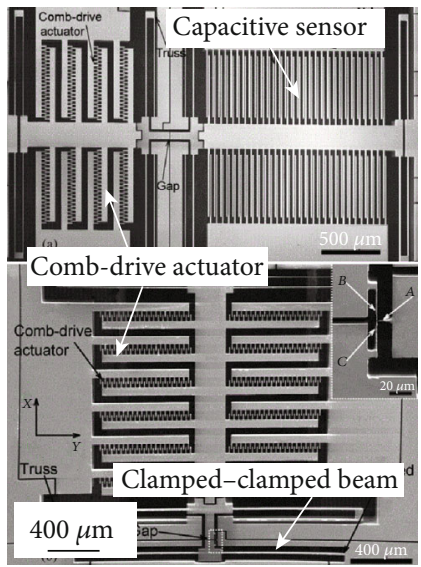

(d)

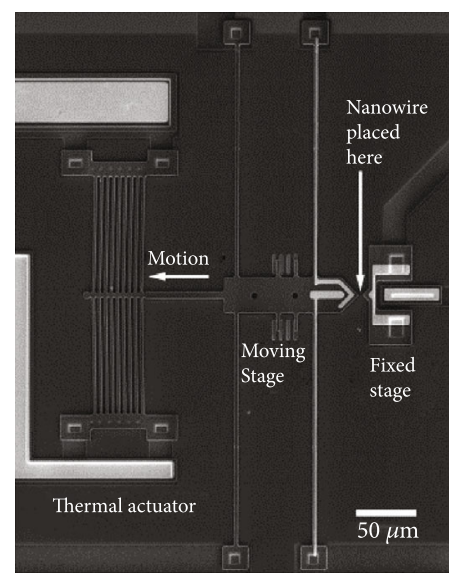

(e)

FIGURE 3: MEMS-based in situ mechanical tensile characterization of nanomaterials in SEM. (a) In situ tensile loading of a templated carbon nanotube (T-CNT). (b) In situ MEMS tensile tester for mechanical characterization of multiwalled nanotubes (MCNTs). (c) FE-SEM micrograph of a carbon nanowire with initial deflection. (d) Two types of nanotensile stages: top picture shows stage with capacitive sensor and bottom one with a clamped-clamped beam force sensor. (e) Microfabricated tensile test structure consisting of electrically isolated moving and fixed stages.

resistance and has been used for quantifying electrical properties of various nanomaterials such as metallic nanowires [80] and carbon nanotubes [81].

Similar with in situ mechanical characterization, CNTs were also frequently adopted as the testing material in the SEM-based in situ electrical characterization. Four-point electrical transport study of single CNT [82] was performed by a combinatory low temperature four-probe scanning tunneling microscope (STM) and SEM, as shown in Figure 4(a). A reliable nanorobotic system consisting of electrothermal microgrippers and mobile microrobots for automated handling and electrical characterization of CNTs was reported in [83].

Except for CNTs, a series of 2D nanomaterials and nanowires were also electrically characterized using fourpoint probing technique. To achieve rapid prototyping of graphene-based devices, a nanorobotic platform was developed for time-saving electrical characterization of graphene [84], where four-point probing of graphene flake was shown in Figure 4(b). Similarly, a four-point probe measurement of individual $\mathrm{SnO}_{2}$ nanowires was achieved by visual servo automated nanomanipulation inside SEM [85], as shown in Figure 4(c).

2.2.2. Two-Point Probing. Compared with four-point probing, there are still experimental scenarios in which fourpoint probing is less feasible for electrical characterization of nanomaterials. For instance, certain types of nanomaterials (e.g., III-nitride nanorods) have relatively low aspect ratios, making it difficult to establish four-point contacts along the sample length. Additionally, to characterize asgrown nanowires vertically attached to their growth substrate, it is more convenient to conduct in situ two-point nanoprobing, with one probe on top of a nanowire and the other on the growth substrate [86].

For CNTs-related two-point electrical characterization, back to 2004, Peng et al. [87] reported a four nanoprobe system in SEM for two-point current-voltage (I-V) measurement of carbon nanotube, as shown in Figure 4(d). Chen et al. [88] obtained linear I-V measurement curves on MCNTs by establishing two-point Ohmic contacts on a CNT using the Joule heating effect. 
TABLE 2: MEMS-based mechanical characterization of micro- and nanomaterials.

\begin{tabular}{|c|c|c|c|}
\hline Testing types & Materials & End-effectors & References \\
\hline \multirow{17}{*}{ Tensile } & & Ims and beams & \\
\hline & Pt films & Thermal actuator & [75] \\
\hline & Freestanding Al films & Electrostatic actuator & {$[62,64]$} \\
\hline & & Bent-beam thermal actuators & [65] \\
\hline & Freestanding PolySi film & Thermal actuator & [59] \\
\hline & Nanocrystalline Ni nanobeam & Thermal actuator & [58] \\
\hline & \multicolumn{3}{|c|}{ CNTs } \\
\hline & MCNTs & Thermal actuator & {$[59,69]$} \\
\hline & & Electrostatic actuator & {$[63,70]$} \\
\hline & T-CNT & Thermal expansion beams & [68] \\
\hline & \multicolumn{3}{|c|}{ 1D nanowires } \\
\hline & Ni nanowires & Nanoindenter head & [66] \\
\hline & Carbon nanowires & Electrostatic actuator & [67] \\
\hline & Co nanowires & Electrostatic actuator & [71] \\
\hline & Si nanowires & Electrostatic actuator & [72] \\
\hline & $\mathrm{ZnO}$ nanowires & Electrostatic actuator & [73] \\
\hline & GaN nanowires & Thermal actuator & {$[74]$} \\
\hline \multirow{3}{*}{ Bending } & Polyacrylonitrile nanofibers & Folded-beam loadcell & {$[76]$} \\
\hline & Microcantilever aluminum beam & Comb-drive probe & [62] \\
\hline & Thin-film polysilicon microstructures & Rotary comb-drive actuator & [77] \\
\hline
\end{tabular}

A bottom-up technique for nanomanipulation combining STM and SEM was proposed in [89]; the author fabricated two nanocontacts at the end of the $\mathrm{GdSi}_{2}$ nanowire and performed direct electrical transport measurement. A metal-semiconductor-metal (M-S-M) model for quantitative analysis of current-voltage characteristics of semiconducting nanowires is proposed in [90], and two-terminal probing was employed for experimental I-V characterization of $\mathrm{Bi}_{2} \mathrm{~S}_{3}$ nanowire transistor. Besides above $1 \mathrm{D}$ nanomaterials, the electrical conductivity of promising 2D MXene nanosheets was demonstrated by the two-point probing method [91].

2.2.3. Three-Point Probing. Three-point field effect measurements were carried out on CNT by Chen et al. [88] using three-point probing technique where a third probe employed as the gate pole, illustrated in Figure 4(e). In situ three-point electrical nanotransport measurements of individual $\mathrm{GdSi}_{2}$ nanowires were carried out to investigate the electrical conductance property, where Au-coated STM tip was employed as the third probe [92], as shown in Figure 4(f). Similarly, to evaluate the electrical characteristics of the isolated $\mathrm{Nb}_{2} \mathrm{Se}_{9}$ flake, electrical transport measurement of single $\mathrm{Nb}_{2} \mathrm{Se}_{9}$ field effect transistor (FET) was conducted using Si tips coated by Cr-Pt [93].

Besides above three types of SEM-based in situ electrical characterization, there are also certain scenarios where SEM was employed as important tool in assisting the probe of advanced electrical properties of nanomaterials. Specifically, taking advantage of electrons' interaction with the sample atoms in SEM, Mayeen et al. [97] investigated the underlying information about the sample's electrical conductivity. To thoroughly understand the reason why such a small amount of nanostructured polyaniline (PANIs) can tremendously enhance the electrical conductivity of the ECCs, SEM was used to characterize the phase separation process and the self-assembly process in electrical conductive composite (ECC) films [45]. In a most recent work [98], a highthroughput electrical characterization of nanotechnology device arrays was enabled by SEM techniques in selection of purely single nanowires. SEM-based in situ characterization techniques could also be applied to tracking the structural reconstruction of the catalysts; in a recent perspective, Zhu et al. [99] established an "in-situ probing map" and offered guidelines for the successful development of nextgeneration efficient electrocatalysts.

2.2.4. ESEM-Based In Situ Electrical Characterization of Biological Cells. The electrical characterization of single cells is challenging cause deep penetration of nanoprobe into the cells will burst it with high stress level risk [21]; this issue was solved by performing short penetration of dual nanoprobe, and the single cell's electrical conductivity was measured [94]. Besides, for the first time, the electrical property of single cells under native condition was reported in [95], where single pulse current measurement was carried out on single cells using dual nanoprobe via a ESEM nanomanipulator system. Also, the electrical response of the human embryonic kidney cell corresponding to external mechanic stimulation was studied by Zhang [96] based on a robotassisted AFM manipulation system. However, there are 


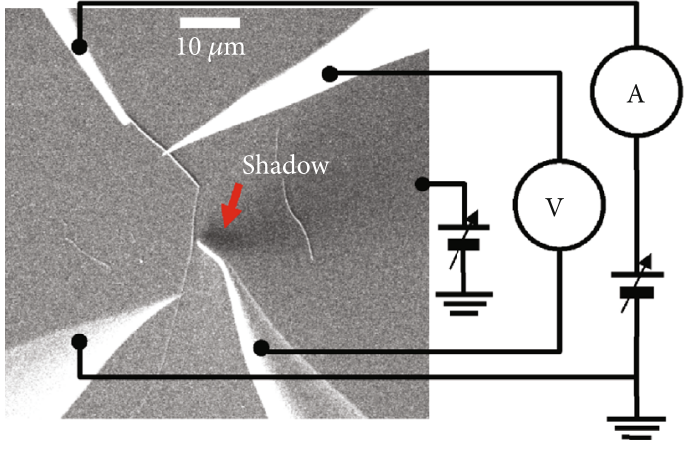

(a)

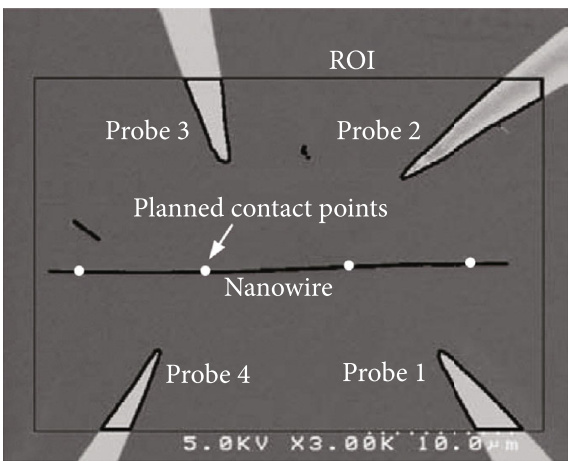

(c)

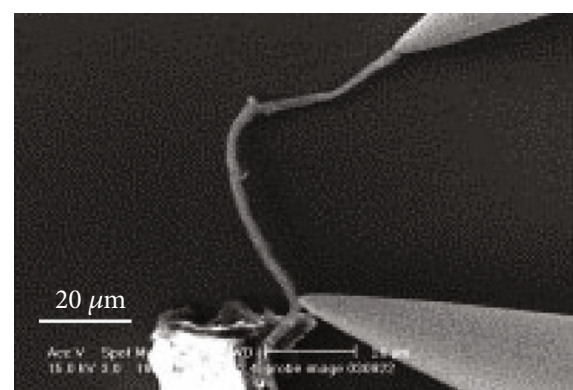

(d)

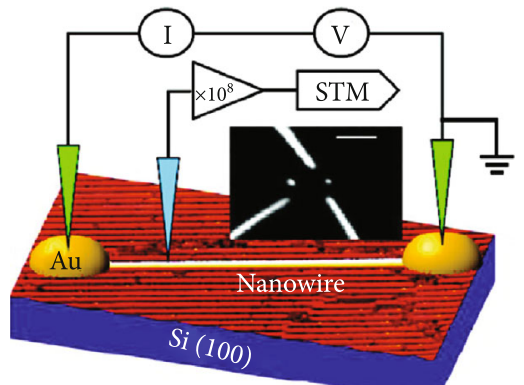

(f)

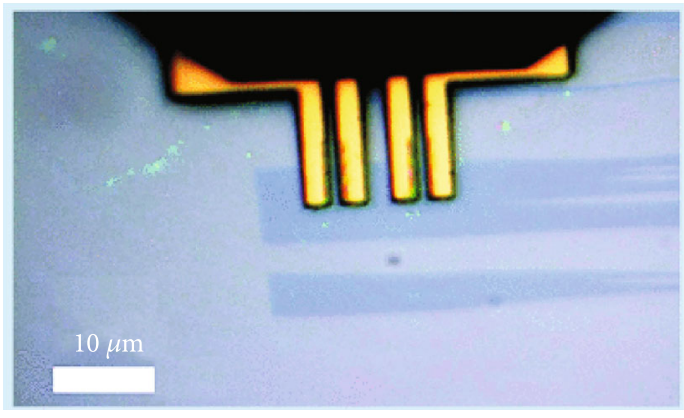

(b)

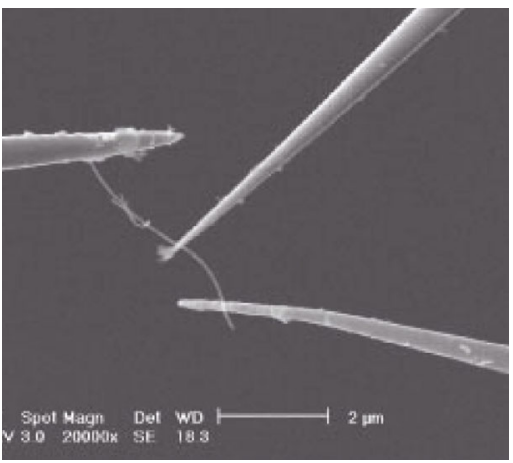

(e)

FIgURE 4: SEM-based in situ electrical characterization of nanomaterials. (a) Four-point electrical transport measurement of single CNT. (b) Four-point probing of graphene flake. (c) Visual servo automated four-point probing of single nanowire. (d) Two-point electrical measurement of single nanotube by using two nanoprobes. (e) Three-point field effect measurements on CNT. (f) Three-point measurement configurations for in situ electrical transport and local density of states on a single $\mathrm{GdSi}_{2}$ nanowire.

still some challenges for electrical characterization of the single cell electrical conductivity, such as limited throughput and sensing ability of robotic manipulation system [22].

Based on above reviews and discussions, the electrical characterization of nanomaterials in SEM is summarized in Table 3, classified by different testing types, categories of nanomaterials, and different kinds of end-effectors.

\subsection{Electromechanical Characterization in SEM. In addition,} the intrinsically coupled electromechanical properties of nanomaterials such as piezo-electrical [100-102] and piezoresistive $[103,104]$ properties have provided special routes of detecting mechanical loading from the electrical change of the nanomaterial and controlling mechanical deformation of nanomaterials via electrical excitation. In the meanwhile, the electromechanical characterization of thin films, nano- wires, and nanobelts benefits their potential applications in biosensor development [100, 105], actuators, and motioncontrollers [78]. Therefore, it is of great interest to carry out dual-field electromechanical characterization of nanomaterials $[106,107]$.

\subsubsection{Electromechanical Characterization of CNT. Tracking} back to the year of 1999, there has been reported work [108] exploring the correlation between mechanical and electrical properties of carbon nanotubes, where carbon nanotubes were stressed while monitoring their conductivity under real-time SEM inspection. Subsequently, the electromechanical characterization of carbon nanotubes $[15,109,110]$ was carried out to investigate the coupling effect between its mechanical and electrical properties, such as the resistance SEM in situ measurement of CNT versus the stress/strain 
TABLE 3: Electrical characterization of nanomaterials in SEM.

(a)

\begin{tabular}{|c|c|c|c|}
\hline Testing types & Nanomaterials & Properties & References \\
\hline \multirow{4}{*}{ Four-point probing } & Boron nanowires & Conductivity & {$[80]$} \\
\hline & CNTs & Current-voltage characteristics & {$[81-83]$} \\
\hline & Graphene flake & Conductance & {$[84]$} \\
\hline & $\mathrm{SnO}_{2}$ nanowires & Current-voltage characteristics & {$[85]$} \\
\hline \multirow{5}{*}{ Two-point probing } & InN nanowires & Electrical transport properties & {$[86]$} \\
\hline & MCNTs & Current-voltage characteristics & {$[87,88]$} \\
\hline & $\mathrm{GdSi}_{2}$ nanowires & Electrical transport properties & [89] \\
\hline & $\mathrm{Bi}_{2} \mathrm{~S}_{3}$ nanowires & Current-voltage characteristics & {$[90]$} \\
\hline & 2D MXene nanosheets & Electrical conductivity & {$[91]$} \\
\hline \multirow{3}{*}{ Three-point probing } & CNT & Field effect measurements & {$[88]$} \\
\hline & $\mathrm{GdSi}_{2}$ nanowires & Electrical conductance property & {$[92]$} \\
\hline & $\mathrm{Nb}_{2} \mathrm{Se}_{9} \mathrm{FET}$ & Electrical transport property & [93] \\
\hline
\end{tabular}

(b) ESEM for biological cells

\begin{tabular}{lccc}
\hline Nanomaterials & Properties & End-effectors & References \\
\hline W303 yeast cells & Single cell electrical conductivity & Dual nanoprobes & [94] \\
W303 yeast cells & Single pulse current measurement & ESEM nanomanipulator system & [95] \\
Human embryonic kidney cell & Current response to indentation force & Robot-assisted AFM manipulation system & [96] \\
\hline
\end{tabular}

property [109] and the electrical properties of various types of suspended single-walled CNTs under the influence of tensile stretching [110], as well as the effects of axial strain on electrical transport properties of individual thin CNTs [15].

2.3.2. Piezoresistance Effect Investigation. The piezoresistance effect of silicon nanowires has also been widely investigated [111] in order to improve the performance of silicon transistors. For the first time, the giant piezoresistance effect in $\mathrm{Si}$ nanowires (as shown in Figure 5(a)) was discovered in [111], which predicted significant perspective in nanowirebased flexible electronics and NEMS. The phenomenon of giant piezoresistance in silicon NWs was further well controlled in [112] for potential application of stress-gated field-effect transistor with a high gauge factor. Anomalous piezoresistance effect [113] was discovered for p-type single crystal silicon nanowires under ultrahigh strain, as illustrated in the SEM image in Figure 5(b). A Si nanowire was suspended in the microelectromechanical testing module. To avoid the effect of electron beam (e-beam) irradiation during nanomaterial testing, Zhang et al. [79] developed a MEMS device for piezoresistivity characterization of synthetic silicon nanowires, where simultaneous electrical and mechanical characterization of individual $\mathrm{Si}$ nanowires could be carried out.

The coupled piezoresistive characterization of some other types of nanostructures has also been carried out through nanomanipulation and electron-beam-induced deposition (EBID) inside SEM. For instance, as shown in Figure 5(c), the conductivity of the deformed nanospring [114] was investigated experimentally; the electromechanical proper- ties of InGaAs/GaAs nanosprings were also characterized in [115], illustrating a potential way to realize electromechanical sensors. The piezoelectric and piezoresistive effects of InAs NWs [116] were studied adopting in situ SEM tensile test method, as shown in Figure 5(d). The superior piezoelectric properties and piezoresistance effect of Sb-doped $\mathrm{ZnO}$ nanobelts were investigated through large longitudinal electromechanical characterization [117], as shown in Figure 5(e). In addition, the piezoresistive response of quasi-1D $\mathrm{ZnO}$ nanowires was characterized using an in situ SEM-based indentation system [118].

\subsubsection{MEMS-Based In Situ Electromechanical Characterization} in SEM. The capability of simultaneous electrical and mechanical measurements of individual nanostructure has demonstrated MEMS devices as a popular platform for piezoresistivity characterization of single nanowires $[79,103,119]$ and nanofiber [105] for the development of novel nanomechanical sensors. A MEMS device was developed for electromechanical characterization of nanowires [79]. Typically, individual nanowires are grown directly between actuators of MEMS device, so that the uniaxial tensile load can be applied to single NWs; for instance, carbon nanowires (CNWs) were fabricated on electrostatically actuated nanotensile tester by FIB-CVD [103]. Cu and SiC nanowires have been integrated into the MEMS tensile testing chip [120], and their mechanical and electronic properties under different stress were characterized simultaneously, as shown in Figure 5(f).

SEM could also be employed as crucial assistance for electromechanical characterization. In the investigation of 


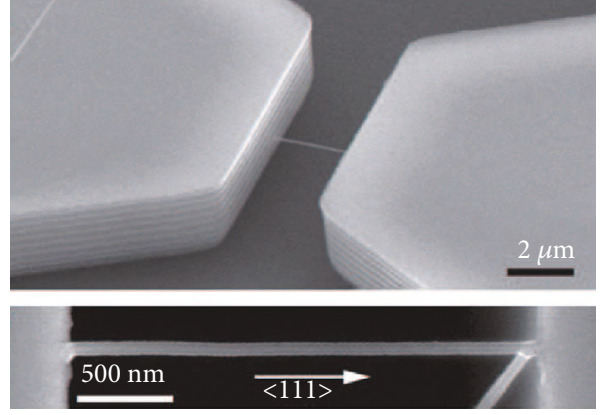

(a)

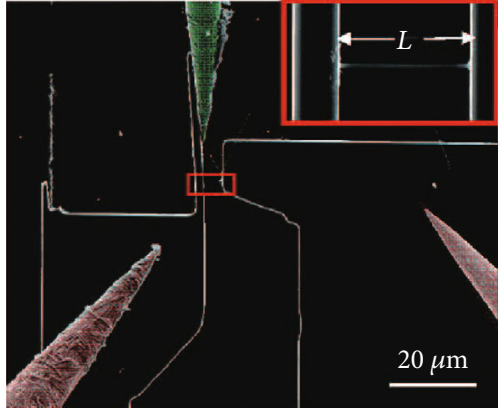

(b)

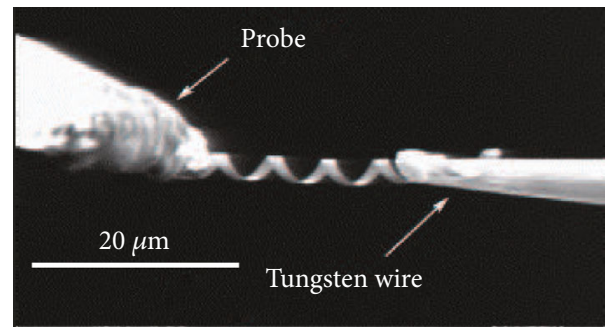

(c)

(e)

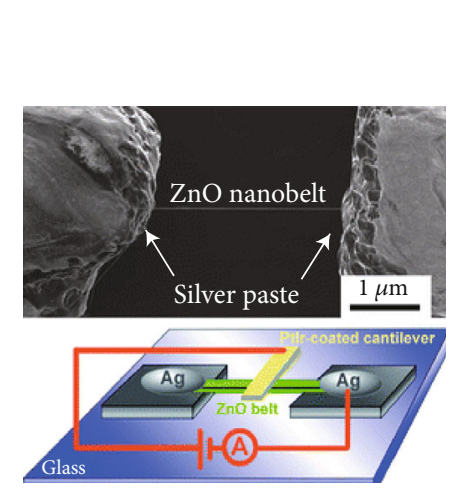

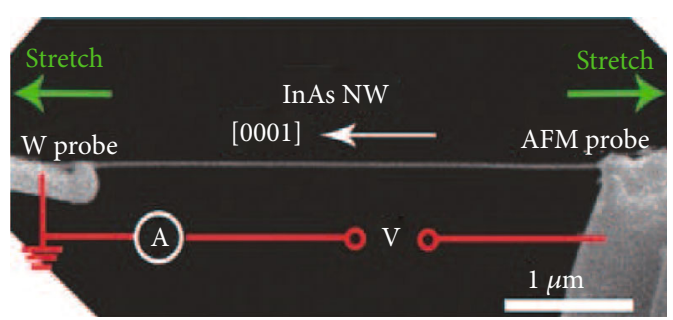

(d)

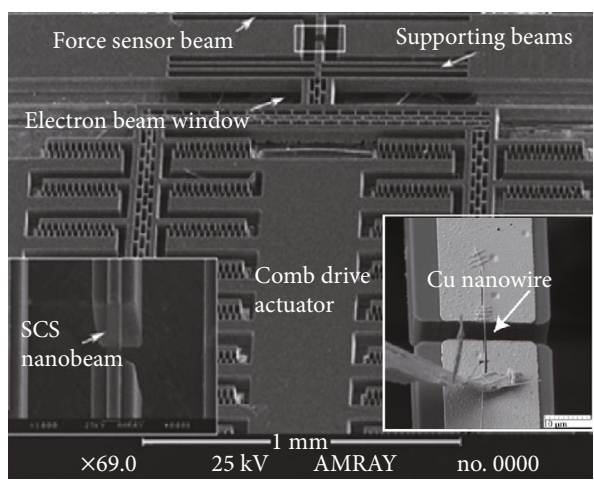

(f)

FIgURE 5: Electromechanical characterization of nanomaterials. (a) Giant piezoresistance effect: single Si nanowires bridged in the trench of SOI substrate. (b) MEMS loading system for tensile testing of a suspended Si nanowire and measuring the resistance change simultaneously. (c) Conductivity measurement of deformed InGaAs/GaAs nanosprings as electromechanical sensor. (d) Electromechanical measurement of an InAs NW using a W probe and an AFM probe, the NW is uniaxially stretched, and its electrical transport properties are measured at different tensile strains. (e) SEM image of a single Sb-doped $\mathrm{ZnO}$ nanobelt fixed by using the silver paste and the schematic diagram of the electromechanical characterization measurements. (f) SEM image of MEMS tensile-testing chip, Cu nanowire is integrated onto the MEMS nanobeam, and its mechanical and electronic properties under different stress were characterized simultaneously.

structure dependent properties of carbon nanomaterials [121], the detailed packing structure and morphology have been revealed through SEM for the following electromechanical measurements. The effect of the morphology (checked by SEM) of the reduced graphene oxide (rGO) powders was studied through electromechanical measurements [122]. The electromechanical properties of the developed nanocomposite-based strain sensors are characterized in detail with the support of the cross-sectional SEM images of the packaged 1D sensing material [123]. In the direct electromechanical characterization of pressuredependent contact current [124], the growth analysis of semiconductor NWs was examined using SEM. The characterization of piezoresistive polyurethane/graphene nanoplatelets coating for strain sensing applications was enabled by the morphological investigations of the produced nanomaterials using FE-SEM [125].

\section{Perspective: Challenges and Future Work}

3.1. Current Status: Few Optical-Related Characterization in SEM. With the rapid advance of optoelectronic devices, the optical and optoelectronic characterization of nanostructures is becoming more and more popular, which benefits the improvement of optoelectronic device as well as the determination of the correlation between properties and the geometrical parameters of nanostructures. However, as an important characterization platform, SEM was not often utilized for pure optical or optoelectronic characterization of nanomaterials, which is partially due to the technical 
challenge of integrating optical components inside the spacelimited SEM chamber and achieving high-efficient optical excitation and measurement in the SEM environment. In the following texts, we will give a short review of opticalmeasurement-related characterization of nanomaterials in SEM and analyze the existing challenges for performing optoelectronic characterization in SEM.

For optical characterization of individual nanostructures, techniques of microphotoluminescence (micro-PL) [126] and scanning near field optical microscopy [126] were often employed in ambient environment. While in SEM, only the cathodoluminescence (CL) characterization of $\mathrm{GaN}$ film [127], GaN crystal [128], and $\mathrm{ZnO}$ columns [129] was reported previously. Also, the photoluminescence (PL) and CL characteristics of $\mathrm{ZnO}$ nanorod [130] were obtained by customized in situ optical characterization system in SEM. Some optoelectronic characterizations were assisted by focused ion beam (FIB) for deposition of metal contacts in an SEM chamber. For instance, the photoconductivity of ZnO NW-based UV photodetectors [131] was characterized with FIB-Pt deposition for electrical contacts.

\subsection{Challenges for Optoelectronic Characterization in SEM}

3.2.1. Contact Resistance. In optoelectronic characterization of nanomaterials, the contact resistance between an electrode/nanoprobe and a sample could significantly affect the measured current-voltage (I-V) data; therefore, it is highly desired to minimize the contact resistance during electrical characterization of nanomaterials. Several studies have been reported for reducing the contact resistance of metal electrodes (formed by EBL or EBID) through rapid thermal annealing [132], electric current flowing [133], e-beam irradiation [134], and EBID [135].

3.2.2. Contact Force/Pressure. During the process of in situ electrical characterization, applying a gentle contact force may ensure a good electrical contact; however, high applied contact forces/pressures pose risks of modifying the electrical properties of tested materials. Chen et al. [136] have investigated the effect of high contact forces/pressures on the modification of electrical properties using scalpel AFM and proposed a process flow to correct the use of scalpel AFM to characterize the electrical properties of nanomaterials in three dimensions.

\subsubsection{Efficient Light Detection in Limited-Size SEM Chamber.} To perform optical characterization in SEM, efficient light collection and detection are necessary. A sizeable paraboloidal mirror was employed in a typical modern setup [137] for light collection in CL test; however, due to the limited space in the SEM chamber, the mirror blocked most other detectors and the electrical nanoprobe integration, thus hindered the simultaneous measurement of electrical and optical properties of nanodevices and nanomaterials. To address this issue, space-saving optical fibers [130] were integrated into the SEM chamber for in situ comprehensive optical characterization of individual optoelectronic nanostructures, providing inspiration for our strategy of optical fiber integration in SEM for optoelectronic characterization.
3.3. Future Work: Demand of Multiphysical Characterization System in SEM. Functional nanomaterials usually have unique multiphysical properties (mechanical, electrical, and optical properties) compared with their bulk counterparts. These multiphysical properties not only exist in independent states but also often coupled with each other and closed correlated as piezoelectric, photoplastic, and optoelectronic properties. For instance, widely studied multifunctional $\mathrm{ZnO}$ NWs possess unique physical characteristics such as semiconductivity and piezoelectricity and have found novel applications in sensors and biomedical science [138]. The observed remarkable photo-induced elastic effect in 1D semiconducting $\mathrm{ZnO}$ nanobelt [139] has demonstrated the significance of mechanical, optical, and electronic coupling in 1D nanostructures. Besides, III-nitride NWs (e.g., InN, $\mathrm{GaN}$, and $\mathrm{AlN}$ ) have been pivotal for optoelectronic applications such as ultrahigh-speed nanoscale lasers and photodetectors, full solar spectrum photovoltaic devices, and high-efficiency white light-emitting diodes (LEDs) [140]. These applications usually require optoelectronic characterization of the III-nitride NWs.

Therefore, for abovementioned nanomaterials, a SEMbased system capable of characterizing the mechanical, electrical, or optical property of nanomaterials independently or simultaneously is highly demanded. SEM-based in situ nanomanipulation technique owns several merits such as ultrahigh positioning resolution, accurately quantified mechanical excitation, and measurement on single nanostructure, as well as the nondestructive electrical nanoprobing capability of nanomaterials; all these merits will contribute to the establishment of SEM-based multiphysical characterization system of nanomaterials.

\section{Conclusion}

This review summarizes existing SEM-based platforms and SEM-enabled techniques for multiphysical (mechanical, electrical, and electromechanical) in situ characterization of nanomaterials. Also, a short review and existing challenges of optical-measurement-related characterization of nanomaterials in SEM are presented. From our perspectives, an SEMbased in situ multiphysical characterization system capable of characterizing the mechanical, electrical, or optical property of nanomaterials is in urgent need, which allows in situ assembly and comprehensive coupled-field nanomaterial characterization (optical, electrical, and mechanical) of individual nanomaterials and enables the systematic investigation of the complicated underlying coupled-field properties of advanced nanomaterials.

\section{Data Availability}

The datasets used or reported in the current work are available from the corresponding author on reasonable request.

\section{Conflicts of Interest}

The authors declare that they have no conflicts of interest. 


\section{Authors' Contributions}

Juntian Qu wrote the manuscript. Xinyu Liu provided insightful suggestions.

\section{Acknowledgments}

This research was supported by the China Postdoctoral Science Foundation Fellowship (No. 2020M680533), the Shuimu Tsinghua Scholar Program (No. 2019SM064), the International Postdoctoral Exchange Fellowship Program (Talent-Introduction Program, No. YJ20190175), and the International Postdoctoral Exchange Fellowship (Academic Exchange Program).

\section{References}

[1] E.-K. Jeon, C.-H. Park, J. A. Lee et al., "Electromechanical properties of single-walled carbon nanotube devices on micromachined cantilevers," Journal of Micromechanics and Microengineering, vol. 22, no. 11, article 115010, 2012.

[2] M. F. L. De Volder, S. H. Tawfick, R. H. Baughman, and A. J. Hart, "Carbon nanotubes: present and future commercial applications," Science, vol. 339, no. 6119, pp. 535-539, 2013.

[3] Y. Li, F. Qian, J. Xiang, and C. M. Lieber, "Nanowire electronic and optoelectronic devices," Materials Today, vol. 9, no. 10, pp. 18-27, 2006.

[4] Z. L. Wang, "Zno nanowire and nanobelt platform for nanotechnology," Materials Science and Engineering: R: Reports, vol. 64, no. 3-4, pp. 33-71, 2009.

[5] C. L. Freeman, F. Claeyssens, N. L. Allan, and J. H. Harding, "Graphitic nanofilms as precursors to wurtzite films: theory," Physical Review Letters, vol. 96, no. 6, article 066102, 2006.

[6] W. Zhao, J. Xu, C. Shi, and H. Chen, "Fabrication, characterization and application of gold nano-structured film," Electrochemistry Communications, vol. 8, no. 5, pp. 773-778, 2006.

[7] Y. Zhang, N. K. Grady, C. Ayala-Orozco, and N. J. Halas, "Three-dimensional nanostructures as highly efficient generators of second harmonic light," Nano Letters, vol. 11, no. 12, pp. 5519-5523, 2011.

[8] C. S. Lao, P. X. Gao, R. S. Yang, Y. Zhang, Y. Dai, and Z. L. Wang, "Formation of double-side teethed nanocombs of $\mathrm{ZnO}$ and self-catalysis of Zn-terminated polar surface," Chemical Physics Letters, vol. 417, no. 4-6, pp. 358-362, 2006.

[9] X. Yang, C. Jin, C. Liang, D. Chen, M. Wu, and J. C. Yu, "Nanoower arrays of rutile tio2," Chemical Communications, vol. 47, pp. 1184-1186, 2011.

[10] S. Choi, H. Lee, R. Ghaffari, T. Hyeon, and D.-H. Kim, "Recent advances in flexible and stretchable bio-electronic devices integrated with nanomaterials," Advanced Materials, vol. 28, no. 22, pp. 4203-4218, 2016.

[11] M. Casini, Smart Buildings: Advanced Materials and Nanotechnology to Improve Energy- Efficiency and Environmental Performance, Woodhead Publishing, 2016.

[12] M. You, Z. Li, P. Zhang, D. Bai, M. Lin, and F. Xu, "Nanomaterial- and micromaterial-based immunoassays," in Handbook of Immunoassay Technologies, S. K. Vashist and J. H. Luong, Eds., pp. 273-304, Academic Press, 2018.

[13] R. K. Debnath, R. Meijers, T. Richter, T. Stoica, R. Calarco, and H. Luth, "Mechanism of molecular beam epitaxy growth of GaN nanowires on Si(111)," Applied Physics Letters, vol. 90, no. 12, article 123117, 2007.

[14] C. Shi, D. K. Luu, Q. Yang et al., "Recent advances in nanorobotic manipulation inside scanning electron microscopes," Microsystems \& Nanoengineering, vol. 2, no. 1, article 16024, 2016.

[15] X. L. Wei, Q. Chen, L. M. Peng, R. Cui, and Y. Li, "In situ measurements on individual thin carbon nanotubes using nanomanipulators inside a scanning electron microscope," Ultramicroscopy, vol. 110, no. 3, pp. 182-189, 2010.

[16] T. Fukuda, M. Nakajima, Pou Liu, and M. R. Ahmad, "Bringing the nanolaboratory inside electron microscopes," IEEE Nanotechnology Magazine, vol. 2, no. 2, pp. 18-31, 2008.

[17] M. F. Chisholm, Scanning Microscopy for Nanotechnology, Springer, 2006.

[18] T. Fukuda, F. Arai, and Lixin Dong, "Assembly of nanodevices with carbon nanotubes through nanorobotic manipulations," Proceedings of the IEEE, vol. 9, no. 11, pp. 1803-1818, 2003.

[19] M. A. Haque, H. D. Espinosa, and H. J. Lee, "MEMS for in situ testing-handling, actuation, loading, and displacement measurements," MRS Bulletin, vol. 35, no. 5, pp. 375-381, 2010.

[20] Y. Zhu and T. H. Chang, "A review of microelectromechanical systems for nanoscale mechanical characterization," Journal of Micromechanics and Microengineering, vol. 25, no. 9, article 093001, 2015.

[21] T. Fukuda, M. Nakajima, M. R. Ahmad, Y. Shen, and M. Kojima, "Micro- and nanomechatronics," IEEE Industrial Electronics Megazine, vol. 4, no. 4, pp. 13-22, 2010.

[22] Y. Shen and T. Fukuda, "State of the art: micro-nanorobotic manipulation in single cell analysis," Robotics and Biomimetics, vol. 1, no. 1, p. 21, 2014.

[23] C. Jiang, Y. Lu, H. Zhang, Y. Shen, and Y. Lu, "Recent advances on in situ SEM mechanical and electrical characterization of low-dimensional nanomaterials," Scanning, vol. 2017, 11 pages, 2017.

[24] M. Minary-Jolandan and M.-F. Yu, "Mechanical and electromechanical characterization of one-dimensional piezoelectric nanomaterials," in Piezoelectric Nanomatersials for Biomedical Applications, G. Ciofani and A. Menciassi, Eds., pp. 6391, Springer, Berlin Heidelberg, 2012.

[25] D. Maharaj and B. Bhushan, "Scale effects of nanomechanical properties and deformation behavior of Au nanoparticle and thin film using depth sensing nanoindentation," Beilstein Journal of Nanotechnology, vol. 5, pp. 822-836, 2014.

[26] R. Agrawal, B. Peng, E. E. Gdoutos, and H. D. Espinosa, "Elasticity size effects in $\mathrm{ZnO}$ nanowires-a combined experimentalcomputational approach," Nano Letters, vol. 8, no. 11, pp. 3668-3674, 2008.

[27] T. Namazu, Y. Isono, and T. Tanaka, "Evaluation of size effect on mechanical properties of single crystal silicon by nanoscale bending test using AFM," Journal of Microelectromechanical Systems, vol. 9, no. 4, pp. 450-459, 2000.

[28] M. F. Pantano, H. D. Espinosa, and L. Pagnotta, "Mechanical characterization of materials at small length scales," Journal of Mechanical Science and Technology, vol. 26, no. 2, pp. 545-561, 2012.

[29] S. Fatikow, V. Eichhorn, F. Krohs, I. Mircea, C. Stolle, and S. Hagemann, "Development of automated microrobot- 
based nanohandling stations for nanocharacterization," Microsystem Technologies, vol. 14, no. 4-5, pp. 463-474, 2008.

[30] M. Nakajima, F. Arai, and T. Fukuda, "In situ measurement of Young's modulus of carbon nanotubes inside a TEM through a hybrid nanorobotic manipulation system," IEEE Transactions on Nanotechnology, vol. 5, no. 3, pp. 243-248, 2006.

[31] M. F. Yu, O. Lourie, M. J. Dyer, K. Moloni, T. F. Kelly, and R. S. Ruoff, "Strength and breaking mechanism of multiwalled carbon nanotubes under tensile load," Science, vol. 287, no. 5453, pp. 637-640, 2000.

[32] D. Bell, L. Dong, B. Nelson, M. Golling, L. Zhang, and D. Grützmacher, "Fabrication and characterization of threedimensional InGaAs/GaAs nanosprings," Nano letters, vol. 6, no. 4, pp. 725-729, 2006.

[33] Y. Zhu, F. Xu, Q. Qin, W. Y. Fung, and W. Lu, "Mechanical properties of vapor-liquid-solid synthesized silicon nanowires," Nano Letters, vol. 9, no. 11, pp. 3934-3939, 2009.

[34] S. T. Boles, A. Sedlmayr, O. Kraft, and R. Mönig, "In situ cycling and mechanical testing of silicon nanowire anodes for lithium-ion battery applications," Applied Physics Letters, vol. 100, no. 24, article 243901, 2012.

[35] Y. Zhu, Q. Qin, F. Xu et al., "Size effects on elasticity, yielding, and fracture of silver nanowires: in situ experiments," Physical Review B, vol. 85, no. 4, pp. 1-7, 2012.

[36] S. Zimmermann, T. Tiemerding, T. Li, W. Wang, Y. Wang, and S. Fatikow, "Automated mechanical characterization of 2-D materials using SEM based visual servoing," International Journal of Optomechatronics, vol. 7, no. 4, pp. 283295, 2013.

[37] S. Zimmermann, V. Eichhorn, and S. Fatikow, "Nanorobotic transfer and characterization of graphene flakes," in IEEE International Conference on Intelligent Robots and Systemspp. 640-645, Algarve, Portugal, 2012.

[38] M. R. Mikczinski, G. Josefsson, G. Chinga-carrasco, E. K. Gamstedt, and S. Fatikow, "Nanorobotic testing to assess the stiffness properties of nanopaper," IEEE Transactions on Robotics, vol. 30, no. 1, pp. 115-119, 2014.

[39] J.-O. Abrahamians, B. Sauvet, J. Polesel-Maris, R. Braive, and S. Regnier, "A nanorobotic system for in situ stiffness measurements on membranes," IEEE Transactions on Robotics, vol. 30, no. 1, pp. 119-124, 2014.

[40] P. Nikaeen, D. Depan, and A. Khattab, "Surface mechanical characterization of carbon nanofiber reinforced low-density polyethylene by nanoindentation and comparison with bulk properties," Nanomaterials, vol. 9, no. 10, p. 1357, 2019.

[41] S. Gantayat, D. Rout, and S. K. Swain, "Carbon nanomaterial-reinforced epoxy composites: a review," Polymer-Plastics Technology and Engineering, vol. 57, no. 1, pp. 1-6, 2018.

[42] K. Akhtar, S. A. Khan, S. B. Khan, and A. M. Asiri, "Scanning electron microscopy: principle and applications in nanomaterials characterization," in Handbook of Materials Characterization, pp. 113-145, Springer International Publishing, Cham, 2018.

[43] A. Zadhoush, R. Reyhani, and M. Naeimirad, "Evaluation of surface modification impact on PP/MWCNT nanocomposites by rheological and mechanical characterization, assisted with morphological image processing," Polymer Composites, vol. 40, no. S1, pp. E501-E510, 2019.

[44] B. Fathi Achachlouei and Y. Zahedi, "Fabrication and characterization of CMC-based nanocomposites reinforced with sodium montmorillonite and $\mathrm{TiO}_{2}$ nanomaterials," Carbohydrate Polymers, vol. 199, pp. 415-425, 2018.

[45] J. Wen, Y. Tian, C. Hao et al., "Fabrication of high performance printed flexible conductors by doping of polyaniline nanomaterials into silver paste," Journal of Materials Chemistry C, vol. 7, no. 5, pp. 1188-1197, 2019.

[46] M. R. Ahmad, M. Nakajima, M. Kojima, S. Kojima, M. Homma, and T. Fukuda, "Nanofork for single cells adhesion measurement via ESEM-nanomanipulator system," IEEE Transactions on NanoBioscience, vol. 11, no. 1, pp. 70-78, 2012.

[47] Y. Shen, M. Nakajima, S. Kojima, M. Homma, M. Kojima, and T. Fukuda, "Single cell adhesion force measurement for cell viability identification using an AFM cantilever-based micro putter," Measurement Science and Technology, vol. 22, no. 11, article 115802, 2011.

[48] Y. Shen, M. Nakajima, S. Kojima, M. Homma, and T. Fukuda, "Study of the time effect on the strength of cell-cell adhesion force by a novel nano-picker," Biochemical and Biophysical Research Communications, vol. 409, no. 2, pp. 160-165, 2011.

[49] M. R. Ahmad, M. Nakajima, S. Kojima, M. Homma, and T. Fukuda, "The effects of cell sizes, environmental conditions, and growth phases on the strength of individual W303 yeast cells inside ESEM," IEEE Transactions on Nanobioscience, vol. 7, no. 3, pp. 185-193, 2008.

[50] Yajing Shen, M. R. Ahmad, M. Nakajima, S. Kojima, M. Homma, and T. Fukuda, "Evaluation of the single yeast cell's adhesion to ITO substrates with various surface energies via ESEM nanorobotic manipulation system," IEEE Transactions on Nanobioscience, vol. 10, no. 4, pp. 217-224, 2011.

[51] M. R. Ahmad, M. Nakajima, S. Kojima, M. Homma, and T. Fukuda, "In situ single cell mechanics characterization of yeast cells using nanoneedles inside environmental SEM," IEEE Transactions on Nanotechnology, vol. 7, no. 5, pp. 607-616, 2008.

[52] M. R. Ahmad, M. Nakajima, S. Kojima, M. Homma, and T. Fukuda, "Buckling nanoneedle for characterizing single cells mechanics inside environmental SEM," IEEE Transactions on Nanotechnology, vol. 10, no. 2, pp. 226-236, 2011.

[53] M. R. Ahmad, M. Nakajima, S. Kojima, M. Homma, and T. Fukuda, "Nanoindentation methods to measure viscoelastic properties of single cells using sharp, flat, and buckling tips inside ESEM," IEEE Transactions on Nanobioscience, vol. 9, no. 1, pp. 12-23, 2010.

[54] H. Liu, J. Wen, Y. Xiao et al., "In situ mechanical characterization of the cell nucleus by atomic force microscopy," ACS Nano, vol. 8, no. 4, pp. 3821-3828, 2014.

[55] Y. Shen, M. Nakajima, Z. Yang et al., "Single cell stiffness measurement at various humidity conditions by nanomanipulation of a nano-needle," Nanotechnology, vol. 24, no. 14, article 145703, 2013.

[56] Y. Shen, M. Nakajima, Z. Zhang, and T. Fukuda, "Dynamic force characterization microscopy based on integrated nanorobotic AFM and SEM system for detachment process study," IEEE/ASME Transactions on Mechatronics, vol. 20, no. 6, pp. 3009-3017, 2015.

[57] G. E. Dieter and D. J. Bacon, Mechanical Metallurgy, McGraw-hill New York, 1986.

[58] B. Pant, B. L. Allen, T. Zhu, K. Gall, and O. N. Pierron, “A versatile microelectromechanical system for nanomechanical 
testing," Applied Physics Letters, vol. 98, no. 5, article 053506, 2011.

[59] Y. Zhu and H. D. Espinosa, "An electromechanical material testing system for in situ electron microscopy and applications," Proceedings of the National Academy of Sciences, vol. 102, no. 41, pp. 14503-14508, 2005.

[60] Y. Isono, "Micro/nano materials testing for reliable design of MEMS/NEMS," in Micro-Nanomechatronics and Human Science, 2004 and The Fourth Symposium MicroNanomechatronics for Information-Based Society, pp. 3338, Nagoya, Japan, 2004.

[61] Y. Zhang, C. Ru, X. Liu et al., "A mems tensile testing device for mechanical characterization of individual nanowires," in Sensors, 2010 IEEE, pp. 2581-2584, Waikoloa, HI, USA, 2010.

[62] M. Haque and M. Saif, "Microscale materials testing using MEMS actuators," Journal of Microelectromechanical Systems, vol. 10, no. 1, pp. 146-152, 2001.

[63] H. D. Espinosa, Yong Zhu, and N. Moldovan, "Design and operation of a MEMS-based material testing system for nanomechanical characterization," Journal of Microelectromechanical Systems, vol. 16, no. 5, pp. 1219-1231, 2007.

[64] M. A. Haque and M. T. A. Saif, "Deformation mechanisms in free-standing nanoscale thin films: a quantitative in situ transmission electron microscope study," Proceedings of the National Academy of Sciences, vol. 101, no. 17, pp. 63356340, 2004.

[65] S. Kumar, M. A. Haque, and H. Gao, "Notch insensitive fracture in nanoscale thin films," Applied Physics Letters, vol. 94, no. 25, article 253104, 2009.

[66] Y. Ganesan, Yang Lu, Cheng Peng, Hao Lu, R. Ballarini, and Jun Lou, "Development and application of a novel microfabricated device for the in situ tensile testing of 1-D nanomaterials," Journal of Microelectromechanical Systems, vol. 19, no. 3, pp. 675-682, 2010.

[67] M. Kiuchi, S. Matsui, and Y. Isono, "Mechanical characteristics of FIB deposited carbon nanowires using an electrostatic actuated nano tensile testing device," Journal of Micro-electromechanical Systems, vol. 16, no. 2, pp. 191201, 2007.

[68] S. Lu, Z. Guo, W. Ding, D. A. Dikin, J. Lee, and R. S. Ruoff, "In situ mechanical testing of templated carbon nanotubes," Review of Scientific Instruments, vol. 77, no. 12, article 125101, 2006.

[69] B. Peng, M. Locascio, P. Zapol et al., "Measurements of nearultimate strength for multiwalled carbon nanotubes and irradiation-induced crosslinking improvements," Nature Nanotechnology, vol. 3, no. 10, pp. 626-631, 2008.

[70] Y. Zhu, N. Moldovan, and H. D. Espinosa, "A microelectromechanical load sensor for in situ electron and X-ray microscopy tensile testing of nanostructures," Applied Physics Letters, vol. 86, no. 1, article 013506, 2005.

[71] D. Zhang, J. M. Breguet, R. Clavel, L. Philippe, I. Utke, and J. Michler, "In situ tensile testing of individual Co nanowires inside a scanning electron microscope," Nanotechnology, vol. 20, no. 36, p. 365706, 2009.

[72] Dongfeng Zhang, J. M. Breguet, R. Clavel, V. Sivakov, S. Christiansen, and J. Michler, "In situ electron microscopy mechanical testing of silicon nanowires using electrostatically actuated tensile stages," Journal of Microelectromechanical Systems, vol. 19, no. 3, pp. 663-674, 2010.
[73] R. Agrawal, B. Peng, and H. D. Espinosa, "Experimentalcomputational investigation of $\mathrm{ZnO}$ nanowires strength and fracture," Nano Letters, vol. 9, no. 12, pp. 4177-4183, 2009.

[74] J. J. Brown, A. I. Baca, K. A. Bertness, D. A. Dikin, R. S. Ruoff, and V. M. Bright, "Tensile measurement of single crystal gallium nitride nanowires on MEMS test stages," Sensors and Actuators A: Physical, vol. 166, no. 2, pp. 177-186, 2011.

[75] K. Abbas, S. Alaie, and Z. C. Leseman, "Design and characterization of a low temperature gradient and large displacement thermal actuators for in situ mechanical testing of nanoscale materials," Journal of Micromechanics and Microengineering, vol. 22, no. 12, article 125027, 2012.

[76] M. Naraghi, T. Ozkan, I. Chasiotis, S. S. Hazra, and M. P. de Boer, "MEMS platform for on-chip nanomechanical experiments with strong and highly ductile nanofibers," Journal of Micromechanics and Microengineering, vol. 20, no. 12, article 125022, 2010

[77] A. Corigliano, F. Cacchione, B. De Masi, and C. Riva, "Onchip electrostatically actuated bending tests for the mechanical characterization of polysilicon at the micro scale," Meccanica, vol. 40, no. 4-6, pp. 485-503, 2005.

[78] F. Yang and J. C.-M. Li, Micro and Nano Mechanical Testing of Materials and Devices, Springer, 2008.

[79] Y. Zhang, X. Liu, C. Ru, Y. L. Zhang, L. Dong, and Y. Sun, "Piezoresistivity characterization of synthetic silicon nanowires using a mems device," Journal of Microelectromechanical Systems, vol. 20, no. 4, pp. 959-967, 2011.

[80] L. Xiao, H. Xiao-Bo, L. Jun-Ling et al., "Four-probe scanning tunnelling microscope with atomic resolution for electrical and electro-optical property measurements of nanosystems," Chinese Physics, vol. 14, no. 8, pp. 1536-1543, 2005.

[81] Q. Chen, S. Wang, and L.-M. Peng, "Establishing Ohmic contacts for in situ current-voltage characteristic measurements on a carbon nanotube inside the scanning electron microscope," Nanotechnology, vol. 17, no. 4, pp. 10871098, 2006.

[82] T. H. Kim, Z. Wang, J. F. Wendelken, H. H. Weitering, W. Li, and A. P. Li, "A cryogenic Quadraprobe scanning tunneling microscope system with fabrication capability for nanotransport research," Review of Scientific Instruments, vol. 78, no. 12, article 123701, 2007.

[83] V. Eichhorn, S. Fatikow, T. Wortmann et al., "NanoLab: a nanorobotic system for automated pick-and-place handling and characterization of CNTs," in Proceedings - IEEE International Conference on Robotics and Automation, pp. 18261831, Kobe, Japan, 2009.

[84] S. Zimmermann, S. A. Garnica Barragan, and S. Fatikow, "Nanorobotic processing of graphene: a platform tailored for rapid prototyping of graphene-based devices," IEEE Nanotechnology Magazine, vol. 8, no. 3, pp. 14-19, 2014.

[85] Changhai Ru, Yong Zhang, Yu Sun et al., “Automated fourpoint probe measurement of nanowires inside a scanning electron microscope," IEEE Transactions on Nanotechnology, vol. 10, no. 4, pp. 674-681, 2011.

[86] S. Zhao, O. Salehzadeh, S. Alagha, K. L. Kavanagh, S. P. Watkins, and Z. Mi, "Probing the electrical transport properties of intrinsic InN nanowires," Applied Physics Letters, vol. 102, no. 7, article 073102, 2013.

[87] L. M. Peng, Q. Chen, X. L. Liang et al., "Performing probe experiments in the SEM," Micron, vol. 35, no. 6, pp. 495502, 2004. 
[88] Q. Chen, S. Wang, and L. M. Peng, "Establishing Ohmic contacts for in situ current-voltage characteristic measurements on a carbon nanotube inside the scanning electron microscope," Nanotechnology, vol. 17, no. 4, pp. 1087-1098, 2006.

[89] S. Qin, T. H. Kim, Z. Wang, and A. P. Li, "Nanomanipulation and nanofabrication with multi-probe scanning tunneling microscope: from individual atoms to nanowires," Review of Scientific Instruments, vol. 83, no. 6, article 063704, 2012.

[90] Z. Zhang, K. Yao, Y. Liu et al., "Quantitative analysis of current-voltage characteristics of semiconducting nanowires: decoupling of contact effects," Advanced Functional Materials, vol. 17, no. 14, pp. 2478-2489, 2007.

[91] A. Feng, T. Hou, Z. Jia, Y. Zhang, F. Zhang, and G. Wu, "Preparation and characterization of epoxy resin filled with Ti3C2Tx MXene nanosheets with excellent electric conductivity," Nanomaterials, vol. 10, no. 1, p. 162, 2020.

[92] S. Qin, T.-H. Kim, Y. Zhang et al., "Correlating electronic transport to atomic structures in self-assembled quantum wires," Nano Letters, vol. 12, no. 2, pp. 938-942, 2012.

[93] B. J. Kim, B. J. Jeong, S. Oh et al., "Mechanical exfoliation and electrical characterization of a one-dimensional Nb2Se9atomic crystal," RSC Advances, vol. 8, no. 66, pp. 3772437728, 2018.

[94] M. R. Ahmad, M. Nakajima, T. Fukuda, S. Kojima, and M. Homma, "Single cells electrical characterizations using nanoprobe via ESEM-nanomanipulator system," in 2009 9th IEEE Conference on Nanotechnology (IEEE-NANO), pp. 589-592, Genoa, Italy, 2009.

[95] M. R. Ahmad, M. Nakajima, M. Kojima, S. Kojima, M. Homma, and T. Fukuda, "Instantaneous and quantitative single cells viability determination using dual nanoprobe inside ESEM," IEEE Transactions on Nanotechnology, vol. 11, no. 2, pp. 298-306, 2012.

[96] C. Zhang, P. Li, L. Liu, Y. Wang, Z. Gao, and G. Li, “Development of mechanostimulated patch-clamp system for cellular physiological study," IEEE/ASME Transactions on Mechatronics, vol. 19, no. 4, pp. 1138-1147, 2014.

[97] A. Mayeen, L. K. Shaji, A. K. Nair, and N. Kalarikkal, "Morphological characterization of nanomaterials," in Characterization of Nanomaterials, pp. 335-364, Woodhead Publishing, 2018.

[98] L. W. Smith, J. O. Batey, J. A. Alexander-Webber et al., "High-throughput electrical characterization of nanomaterials from room to cryogenic temperatures," ACS Nano, vol. 14, no. 11, pp. 15293-15305, 2020.

[99] Y. Zhu, J. Wang, H. Chu, Y.-C. Chu, and H. M. Chen, "In situ/operando studies for designing next-generation electrocatalysts," ACS Energy Letters, vol. 5, no. 4, pp. 1281-1291, 2020.

[100] R. Zhu, D. Wang, S. Xiang, Z. Zhou, and X. Ye, "Piezoelectric characterization of a single zinc oxide nanowire using a nanoelectromechanical oscillator," Nanotechnology, vol. 19, no. 28, article 285712, 2008.

[101] Y. Zhang, X. Yan, Y. Yang, Y. Huang, Q. Liao, and J. Qi, "Scanning probe study on the piezotronic effect in $\mathrm{ZnO}$ nanomaterials and nanodevices," Advanced Materials, vol. 24, no. 34, pp. 4647-4655, 2012.

[102] X. Li, M. Sun, X. Wei, C. Shan, and Q. Chen, “1D piezoelectric material based nanogenerators: methods, materials and property optimization," Nanomaterials, vol. 8, no. 4, p. 188, 2018.
[103] M. Kiuchi, S. Matsui, and Y. Isono, "The piezoresistance effect of FIB-deposited carbon nanowires under severe strain," Journal of Micromechanics and Microengineering, vol. 18, no. 6, article 065011, 2008.

[104] R. J. Grow, Q. Wang, J. Cao, D. Wang, and H. Dai, “Piezoresistance of carbon nanotubes on deformable thin-film membranes," Applied Physics Letters, vol. 86, no. 9, 2005.

[105] J. J. Brown, J. W. Suk, G. Singh et al., "Microsystem for nanofiber electromechanical measurements," Sensors and Actuators, A: Physical, vol. 155, no. 1, pp. 1-7, 2009.

[106] J. H. Han and M. T. A. Saif, "In situ microtensile stage for electromechanical characterization of nanoscale freestanding films," Review of Scientific Instruments, vol. 77, no. 4, article 045102, 2006.

[107] A. Jourdain, P. D. Moor, K. Baert, I. D. Wolf, and H. A. C. Tilmans, "Mechanical and electrical characterization of BCB as a bond and seal material for cavities housing (RF-)MEMS devices," Journal of Micromechanics and Microengineering, vol. 15, no. 7, pp. S89-S96, 2005.

[108] M. F. Yu, M. J. Dyer, G. D. Skidmore et al., “Three-dimensional manipulation of carbon nanotubes under a scanning electron microscope," Nanotechnology, vol. 10, no. 3, pp. 244-252, 1999.

[109] P. A. Williams, S. J. Papadakis, M. R. Falvo et al., "Controlled placement of an individual carbon nanotube onto a microelectromechanical structure," Applied Physics Letters, vol. 80, no. 14, pp. 2574-2576, 2002.

[110] J. Cao, Q. Wang, and H. Dai, "Electromechanical properties of metallic, quasimetallic, and semiconducting carbon nanotubes under stretching," Physical Review Letters, vol. 90, no. 15, p. 4, 2003.

[111] R. He and P. Yang, "Giant piezoresistance effect in silicon nanowires," Nature Nanotechnology, vol. 1, no. 1, pp. 4246, 2006.

[112] P. Neuzil, C. C. Wong, and J. Reboud, "Electrically controlled giant piezoresistance in silicon nanowires," Nano Letters, vol. 10, no. 4, pp. 1248-1252, 2010.

[113] A. Lugstein, M. Steinmair, A. Steiger, H. Kosina, and E. Bertagnolli, "Anomalous piezoresistance effect in ultrastrained silicon nanowires," Nano Letters, vol. 10, no. 8, pp. 3204-3208, 2010.

[114] D. J. Bell, Y. Sun, L. Zhang, L. X. Dong, B. J. Nelson, and D. Grützmacher, "Three-dimensional nanosprings for electromechanical sensors," Sensors and Actuators A: Physical, vol. 130-131, pp. 54-61, 2006.

[115] G. Hwang, H. Hashimoto, D. J. Bell, L. Dong, B. J. Nelson, and S. Schön, "Piezoresistive InGaAs/GaAs nanosprings with metal connectors," Nano letters, vol. 9, no. 2, pp. 554-561, 2009.

[116] X. Li, X. Wei, T. Xu, D. Pan, J. Zhao, and Q. Chen, "Remarkable and crystal-structure-dependent piezoelectric and piezoresistive effects of InAs nanowires," Advanced Materials, vol. 27, no. 18, pp. 2852-2858, 2015.

[117] Y. Yang, J. Qi, W. Guo, Q. Liao, and Y. Zhang, "Mechanical and longitudinal electromechanical properties of Sb-doped ZnO nanobelts," CrystEngComm, vol. 12, pp. 2005-2007, 2010.

[118] S. Kaps, S. Bhowmick, J. Grottrup et al., "Piezoresistive response of quasione- dimensional $\mathrm{ZnO}$ nanowires using an in situ electromechanical device," ACS Omega, vol. 2, no. 6, pp. 2985-2993, 2017. 
[119] X. Ye, Y. Zhang, R. Changhai, J. Luo, S. Xie, and S. Yu, “Automated pick-place of silicon nanowires," IEEE Transactions on Automation Science and Engineering, vol. 10, no. 3, pp. 554561, 2013.

[120] Y. Wang, T. Li, X. Zhang, H. Zeng, and Q. Jin, "In situ TEM/SEM electronic/mechanical characterization of nano material with MEMS chip," Journal of Semiconductors, vol. 35, no. 8, article 081001, 2014.

[121] G. Wang, Y. Wang, P. Zhang et al., "Structure dependent properties of carbon nanomaterials enabled fiber sensors for in situ monitoring of composites," Composite Structures, vol. 195, pp. 36-44, 2018.

[122] H. Park, S. Lim, D. D. Nguyen, and J. W. Suk, "Electrical measurements of thermally reduced graphene oxide powders under pressure," Nanomaterials, vol. 9, no. 10, 2019.

[123] A. S. Dahiya, T. Gil, J. Thireau et al., "1D nanomaterial-based highly stretchable strain sensors for human movement monitoring and humancrobotic interactive systems," Advanced Electronic Materials, vol. 6, no. 10, article 2000547, 2020.

[124] Y. Calahorra, W. Kim, J. Vukajlovic-Plestina, A. F. i Morral, and S. Kar-Narayan, "Time-resolved open-circuit conductive atomic force microscopy for direct electromechanical characterisation," Nanotechnology, vol. 31, no. 40, article 404003, 2020.

[125] M. Fortunato, I. Bellagamba, F. Marra, A. Tamburrano, and M. S. Sarto, "Development and characterization of a piezoresistive polyurethane/GNP coating for strain sensing applications," in 2020 IEEE 20th International Conference on Nanotechnology (IEEE-NANO), pp. 176-179, Montreal, QC, Canada, 2020.

[126] A. Gustafsson, M.-E. Pistol, L. Montelius, and L. Samuelson, "Local probe techniques for luminescence studies of lowdimensional semiconductor structures," Journal of Applied Physics, vol. 84, no. 4, pp. 1715-1775, 1998.

[127] L. J. Brillson, "Nanoscale luminescence spectroscopy of defects at buried interfaces and ultrathin films," Journal of Vacuum Science \& Technology B: Microelectronics and Nanometer Structures Processing, Measurement, and Phenomena, vol. 19, no. 5, pp. 1762-1768, 2001.

[128] J. H. Choi, H. Y. Ahn, Y. S. Lee et al., "GaN light-emitting diodes on glass substrates with enhanced electroluminescence," Journal of Materials Chemistry, vol. 22, pp. 2294222948, 2012.

[129] J. B. Baxter, F. Wu, and E. S. Aydil, "Growth mechanism and characterization of zinc oxide hexagonal columns," Applied Physics Letters, vol. 83, no. 18, pp. 3797-3799, 2003.

[130] C. Li, M. Gao, C. Ding et al., "In situ comprehensive characterization of optoelectronic nanomaterials for device purposes," Nanotechnology, vol. 20, no. 17, article 175703, 2009.

[131] J. H. He, P. H. Chang, C. Y. Chen, and K. T. Tsai, "Electrical and optoelectronic characterization of a $\mathrm{ZnO}$ nanowire contacted by focused-ion-beam-deposited Pt," Nanotechnology, vol. 20, no. 13, article 135701, 2009.

[132] J.-O. Lee, C. Park, J.-J. Kim, J. Kim, J. W. Park, and K.-H. Yoo, "Formation of low-resistance ohmic contacts between carbon nanotube and metal electrodes by a rapid thermal annealing method," Journal of Physics D: Applied Physics, vol. 33, no. 16, p. 1953, 2000.

[133] H. Maki, M. Suzuki, and K. Ishibashi, "Local change of carbon nanotube-metal contacts by current flow through elec- trodes," Japanese journal of applied physics, vol. 43, no. 4S, article 2027, 2004.

[134] A. Bachtold, M. Henny, C. Terrier et al., "Contacting carbon nanotubes selectively with low-ohmic contacts for four-probe electric measurements," Applied Physics Letters, vol. 73, no. 2, pp. 274-276, 1998.

[135] N. Yu, M. Nakajima, Q. Shi et al., "Characterization of the resistance and force of a carbon nanotube/metal side contact by nanomanipulation,” Scanning, vol. 2017, 11 pages, 2017.

[136] S. Chen, L. Jiang, M. Buckwell et al., "On the limits of scalpel AFM for the 3D electrical characterization of nanomaterials," Advanced Functional Materials, vol. 28, no. 52, article 1802266, 2018.

[137] B. G. Yacobi and D. B. Holt, "Cathodoluminescence scanning electron microscopy of semiconductors," Journal of Applied Physics, vol. 59, no. 4, pp. R1-R24, 1986.

[138] A. Tuantranont, "Applications of nanomaterials in sensors and diagnostics," in Springer series on chemical sensors and biosensors, vol. 14, Springer, Berlin Heidelberg, 2013.

[139] A. San-Miguel, "Nanomaterials under high-pressure," Chemical Society Reviews, vol. 35, pp. 876-889, 2006.

[140] C. P. Wang, C. W. Liu, and C. Gau, "Silicon nanowire temperature sensor and its characteristic," in 2011 6th IEEE International Conference on Nano/Micro Engineered and Molecular Systems, pp. 630-633, Kaohsiung, Taiwan, 2011. 